\title{
A ANÁLISE DA ESTRUTURA DE UMA POPULAÇÃO DE Lychnophora pinaster Mart. DE UM CAMPO RUPESTRE DO SUL DE MINAS GERAIS, BRASIL, POR MEIO DA ESTATÍSTICA MULTIVARIADA
}

\author{
STRUCTURE ANALYSIS OF A POPULATION OF Lychnophora pinaster Mart., IN \\ CAMPO RUPESTRE, SOUTHERN MINAS GERAIS, BRAZIL, BY MEANS OF \\ MULTIVARIATE STATISTICAL
}

\section{ANÁLISIS DE LA ESTRUCTURA DE UNA POBLACIÓN DE Lychnophora pinaster Mart. EN CAMPO DE RUPESTRE, SUR DE MINAS GERAIS, BRASIL, POR ESTADÍSTICA MULTIVARIANTE}

\author{
Écio Souza Diniz ${ }^{1}$, Ana Paula Pavanelli ${ }^{2}$ y Flávio José Soares Júnior ${ }^{3}$
}

\begin{abstract}
Resumo
O Cerrado brasileiro é caracterizado pelo alto endemismo de taxa botânico; muitos, de reconhecida importância fitoterápica e ou econômica. Um taxon que se destaca por essas condições, Lychnophora pinaster Mart. (Arnica-brasileira) é amplamente explorado por suas qualidades terapêuticas. Assim, com o presente estudo, teve-se o objetivo de elucidar as estratégias de ocupação do espaço físico por uma população de L. pinaster, analisando os parâmetros tradicionais de estrutura por métodos de Análises Multivariadas. Para tanto, foram alocadas 30 parcelas contíguas de 10mx10m em um trecho de Campo Rupestre, dentro das quais, foram medidos os DAS e as alturas de todas as plantas da referida espécie. Os parâmetros estruturais obtidos para a população foram tratados pelo Coeficiente de Distância Euclidiana Simples e, em seguida, analisados por UPGMA, Ligação Completa, e PCoA. As Análises de Agrupamento mostraram consistência quanto aos grandes grupos, estabelecidos em altos níveis de fusão. Dentre as análises feitas, a UPGMA sem a variável "volume" (C.Cf. $=0.884539)$ determinou seis grandes grupos ao nível de fusão 20, com três deles se destacando pelas diferenças de abundância, médias de altura e de diâmetro, por parcela. Neste caso, a Análise de Ordenação revelou 74,94\% da variância acumulada em um eixo. Por fim, a Análise de Agrupamento por Ligação Completa para “perfilhos” (C.Cf.=0.730747), evidenciou dois grandes grupos ao nível de fusão 17, determinados pela distribuição irregular dos indivíduos não perfilhados pelas parcelas. Distribuição esta que, certamente, reflete as condições acidentadas da topografia e dos afloramentos rochosos da área de estudo.
\end{abstract}

Palavras-chave: Arnica, Cerrado, Agrupamento, Ordenação.

\begin{abstract}
The brazilian Cerrado is characterized by high endemism of botanic taxa; many of recognized herbal and or economic importance. A taxon that stands out for these conditions, Lychnophora pinaster Mart. (Arnica-brazilian) is widely exploited for its therapeutic qualities. Thus, the present study was undertaken to elucidate the strategies of occupation of space by a population of $L$. pinaster, analyzing the traditional parameters of structure in multivariate analysis. For this purpose, 30 plots contiguous of $10 \mathrm{mx} 10 \mathrm{~m}$ were allocated in a Campo Rupestre stretch, inside of which DAS and the heights of all plants of that species were measured. The structural parameters obtained for the population were treated by simple Euclidean distance coefficient and then analyzed by UPGMA, complete linkage, and PCoA. Cluster Analyses showed consistency in large groups, set up in high levels of fusion. Among the tests performed, the UPGMA without the variable "volume" (C.Cf.=0.884539) determined six major groups to the fusion level 20, with three of them standing out by the differences in abundance, average height and diameter per plot. In this case, the ordination analysis revealed $74.94 \%$ of variance accumulated on an axis. Finally, Cluster Analysis by Complete Link for "tiller" (C.Cf. $=0.730747)$, showed two major groups at fusion 17, determined by the irregular distribution of plots by individuals no affiliated. This distribution certainly reflects the conditions of the hilly topography and rock outcrops within the study area.
\end{abstract} Key words: Arnica, Cerrado, Cluster, Ordination. 


\begin{abstract}
Resumen
El Cerrado brasileño se caracteriza por la alta tasa de endemismo botánico, y por la importancia económica y medicinal de algunas hierbas como el taxón Lychnophora pinaster Mart. (Arnicabrasileña) que es ampliamente explotada por sus cualidades terapéuticas. El presente estudio fue llevado a cabo para dilucidar las estrategias de ocupación del espacio de una población de $L$. pinaster, con análisis de los parámetros tradicionales de la estructura por análisis multivariado. Para esto, 30 parcelas fueron distribuidas en un tramo contiguo de $10 \mathrm{~m} \mathrm{x} 10 \mathrm{~m}$ en Campo Rupestre, las cuales se midieron el DAS y las alturas de todas las plantas de la especie. Los parámetros estructurales obtenidos en la población fueron tratados por coeficiente simples de distancia euclídeana y luego analizados por UPGMA y ACOP. Los análisis mostraron la consistencia de clúster en grupos grandes, creada en los altos niveles de fusión. El UPGMA sin la variable "volumen" (= 0.884539 C.Cf.) determinó seis grupos principales con el nivel de fusión 20, donde tres de ellos se destacan por las diferencias en la abundancia, la altura y el diámetro promedio por parcela. En este caso, el análisis de ordenación reveló 74.94 \% de la varianza acumulada en un eje. Por último, el análisis de clasificación por Ligación Completa para "división del tallo principal" (= 0.730747 C.Cf.), mostró dos grupos principales en Fusión 17, determinada por la distribución irregular de las parcelas por individuos sin división del tallo. La distribución refleja las condiciones de la topografía accidentada y afloramientos rocosos en el área de estudio.

Palabras claves: Arnica, Savannah, Agrupamientos, Ordenación.
\end{abstract}

\section{Introdução.}

A Savana, uma das formações vegetacionais mais antigas da Terra, é composta por árvores e arbustos espalhados acima de um estrato graminoso onde não há formação de dossel contínuo. Para a América do Sul, as vegetações savânicas são datadas a partir do Terciário (Van Der Hammen, 1983); sendo encontradas na Bolívia, Brasil, Paraguai e na Venezuela, porém com baixa riqueza (Rizzini, 1992; Ratter et al., 1997). No Brasil, as formações savânicas estão representadas pelo Cerrados Denso, Típico ou Stricto Sensu, Ralo e Rupestre; Vereda, Parque de Cerrado e Palmeiral (Ribeiro et al., 1983; Ribeiro \& Walter, 1998).

O Bioma do Cerrado é provavelmente um dos mais ricos e a maior savana do mundo, em função do seu alto endemismo (Myers et al., 2000) e por cobrir, originalmente, cerca de dois milhões de $\mathrm{km}^{2}$ do Brasil, respectivamente. Tal cobertura lhe confere o posto de $2^{\circ}$ maior Bioma do Brasil (Ratter et al., 1997). Sua extensão territorial possui uma área core localizada no Planalto Central brasileiro entre outras áreas adjacentes, sendo algumas delas, áreas disjuntas (Rizzini, 1992). Trechos de Cerrado são encontrados do limite Norte da Floresta Amazônica até o sul do Estado de São Paulo e norte do Paraná, ocupando mais de $20^{\circ}$ de latitude e atingindo altitudes próximas de 1.800 metros.

No Estado de Minas Gerais, o Cerrado é predominante dentre todos os tipos de vegetação existentes. São aproximadamente $53 \%$ de área savânica localizada no oeste do estado e algumas áreas disjuntas a sudeste e sul. Neste Estado, os Campos Cerrados constituem a fisionomia vegetal predominante nas encostas de até 900 metros de altitude, cedendo lugar aos Campos Rupestres e de
Altitude nos trechos mais elevados (Rodrigues \& Carvalho, 2001).

O Campo Rupestre ou Refúgio Vegetacional (Veloso el al., 1991), é uma formação vegetal altamente especializada que ocorre no alto das montanhas das regiões Sudeste, Centro-Oeste e Nordeste, do Brasil. Sua ocorrência está associada a solos rasos ou afloramentos rochosos, com predominância de rochas quartizíticas e arenitos (Harley, 1995). Estes afloramentos rochosos abrigam plantas de diversos portes, desde pequenas árvores até ervas; como por exemplo, as pertencentes à família Asteraceae (Pereira, 1994; Safford, 1999).

A flora dos Campos Rupestres é caracterizada pela presença de espécies distribuídas ecológica e geograficamente, de forma muito restrita; com muitas delas sendo endêmicas destas áreas serranas ou montanhosas (Oliveira-Filho \& Fluminhan-Filho, 1999). Tal vegetação, atualmente, encontra-se bastante ameaçada. Muitas das suas espécies nativas, de importância comercial e ecológica, vêm desaparecendo, devido à ocupação desordenada promovida pela expansão urbana e agropecuária, exploração irracional e uso indiscriminado do fogo (Fielder et al., 2004).

Dentre as várias espécies endêmicas dos Campos Rupestres ameaçadas de extinção, Lychnophora pinaster, conhecida como "Arnica-mineira”, tem uma grande importância ecológica e econômica, ocupando lugar importante na medicina popular e na indústria de fitoterápicos (Souza et al., 2003). No entanto, da mesma maneira que ocorrem com as demais plantas medicinais, os recursos genéticos da Arnica-mineira são obtidos por meio de processo extrativista, muitas vezes sem uma reposição mínima da população, agravando a ameaça de extinção da espécie. 
Uma das formas de se compreender a estrutura e a dinâmica de uma população vegetal investigada é conhecendo a maneira como os seus indivíduos se distribuem por uma comunidade (Hay et al., 2000). Complementando este fato, Harper (1977) justifica a importância do estudo de populações por este fornecer informações básicas ao nível específico; principalmente, relativas à variação no número de indivíduos na população.

Assim, entendendo que os estudos populacionais constituem a principal ferramenta para compreender a dinâmica destas formações savânicas; que o Bioma Cerrado é considerado um dos hot spot da biodiversidade; e visando subsidiar estudos que visem a conservação e ou preservação dos Campos Rupestres e de seus componentes biológicos, desenvolveu-se o presente estudo. Com ele teve-se por objetivos: elucidar as estratégias de ocupação do espaço físico por L. pinaster em um trecho de Campo Rupestre do Sul de Minas Gerais, analisando os parâmetros de estrutura através dos Métodos de Análises Estatísticas de Multivariadas.

\section{Material e métodos.}

\section{Área de estudo.}

Este estudo foi conduzido em uma área de Campo Rupestre da Reserva Biológica UNILAVRASBoqueirão (ReBio Boqueirão) (Figura 1a), propriedade do Centro Universitário de Lavras (UNILAVRAS). A referida Reserva está localizada no Município de Ingaí, sul do Estado de Minas Gerais, às coordenadas $21^{\circ} 14^{\prime}$ 59”'S e $44^{\circ} 59^{\prime}$ 27'W. As altitudes variam em torno dos 1070 metros de altitude.

A ReBio UNILAVRAS-Boqueirão encontra-se na região do Alto Rio Grande, que está constituída atualmente por 26 municípios, incluindo Lavras (o maior dentre os que se localizam próximos a Ingaí) com $537 \mathrm{~km}^{2}$ de extensão territorial, (Rodrigues \& Carvalho, 2001). O Rio Grande, com uma bacia de drenagem de $86800 \mathrm{~km}^{2}$ e $1930 \mathrm{~km}$ de extensão (CETEC, 1983), é barrado no decorrer do seu curso pelas Usinas Hidroelétricas do Funil, Itutinga e Camargos, que representam grandes fontes de energia para os municípios dessa região.

As normais climatológicas caracterizam o Clima da região de Lavras como sendo do tipo Cwb (mesotérmico com verões chuvosos e invernos secos), de acordo com o Sistema de Classificação Climática de Köppen (Eidt, 1968). A temperatura média anual é de $20.4^{\circ} \mathrm{C}$, aumentando para $22.8^{\circ} \mathrm{C}$ nos meses mais quentes; com precipitação média anual de $1460 \mathrm{~mm}$ (Dantas et al., 2007). Dentre os tipos pedológicos comuns a este município, destacam-se os solos Litólicos, Cambissolos, Podzólicos VermelhoAmarelos, Latossolos Vermelho-amarelos, Solos Hidromórficos e Solos Aluviais (Rodrigues \& Carvalho, 2001).
A vegetação da maior parte da região do Alto Rio Grande é representada por fisionomias do Cerrado, como os Campos Cerrados, Matas de Galerias, Matas Ciliares e Campos Rupestres (Queiroz et al., 1980). $\mathrm{Na}$ ReBio UNILAVRAS-Boqueirão, entretanto, podem ser encontradas as fitofisionomias campestres de Cerrado e Campos rupestres, além das Florestas de Galeria e Ciliar (Magalhães et al., 2008; Alexandre Júnior \& Soares Júnior, 2009).

A espécie estudada.

A espécie Lychnophora pinaster (Mart.) (Figura 1b), popularmente conhecida como "Arnica Mineira" (Semir, 1991) é uma espécie perenifólia com comportamento fenológico sazonal (Silva, 1998). Pertencente a família Asteraceae (ou Compositae) (Coile \& Jones, 1981; Semir, 1991), inclui plantas que variam de subarbustos eretos, com muitos ramos, a pequenos arbustos ericóides (Semir, 1991), estando restritas a algumas localidades de Minas Gerais, principalmente aquelas de ambientes xéricos, como os Campos Rupestres, Campos Sujos e Campos Limpos de Altitudes das regiões de Cerrado do Alto Rio Grande (Rodrigues \& Carvalho, 2001).

A Arnica é amplamente utilizada pela medicina tradicional, indústrias cosméticas e farmacêuticas, devido as características de suas folhas e flores aromáticas. Além da sua utilização na forma de extrato alcoólico ou pomada, potencializando suas propriedades anestésica, antiinflamatória e cicatrizante (Souza et al., 2003), também é usada como sabonete para tratar de asperezas e rachaduras na pele (Almeida et al., 1998). Essa ampla aptidão certamente motivou a sua extração predatória, resultando na colocação de seu nome na lista das plantas consideradas vulneráveis a extinção do Estado de Minas Gerais (COPAM, 2009); merecendo, deste modo, um maior número de estudos acerca de sua ecologia que subsidiem novas técnicas para sua conservação.

Procedimentos.

Para o desenvolvimento do presente estudo foi necessário alocar 30 parcelas contíguas, conforme proposto por Greig-Smith (1964), de 10 por 10 metros cada, totalizando $3000 \mathrm{~m}^{2}$ de área amostrada. Os indivíduos presentes em cada parcela foram avaliados quanto à presença de perfilhos, em perfilhados ou não perfilhados; assim, como tiveram suas alturas e DAS (Diâmetro do Tronco medido à Altura do Solo) medidos por meio de uma trena de 10 metros e um paquímetro digital, respectivamente.

Por fim, os parâmetros estruturais usuais calculados, como densidade, altura, diâmetro e volume, foram avaliados por meio das Análises de Agrupamento e de Ordenação. Para tanto, foram montadas matrizes com o resultado das Análises por Coeficiente de Distância Euclidiana Simples, que foram, em seguida, analisadas pelos Métodos de Agrupamento UPGMA (Análise por Médias de Grupos não Ponderadas), Ligação Simples e Ligação 
Completa. A mesma matriz também foi analisada por PCoA (Análise em Coordenadas Principais), para evidenciar os fatores que determinaram a formação dos grupos.

\section{Resultados e discussões.}

A população, dentro da área amostrada, estava formada por 609 indivíduos de Lychnophora pinaster, entre jovens e adultos, de diversos tamanhos. Inicialmente as análises de agrupamento mostraram consistência na formação dos grandes grupos, estabelecidos em altos níveis de fusão.

De acordo com Ferreira (1996), as análises de agrupamento são baseadas na similaridade ou dissimilaridade (distância) entre grupos, sendo que a similaridade entre estes consiste na falta de independência entre eles. A confiabilidade na análise por UPGMA foi estabelecida em função de a mesma ter alcançado o maior valor de Correlação Cofenética (C.Cf.=0.984252 para análise incluindo o parâmetro volume e 0.884539 sem o mesmo) em relação aos métodos de Ligação Simples e Completa.

Com a linha fênon em nível de fusão 400, ficam evidentes três grupos (Figura 2). Destes, o primeiro, constituído apenas pela parcela quatro, se formou no mais alto nível de fusão (1600), mostrando claramente ser a parcela mais distinta de toda a amostragem. $\mathrm{O}$ segundo grupo, formado pelas parcelas um e 23 , e o terceiro grupo, formado pelas demais parcelas, foram estabelecidos em níveis de fusão baixos, como evidência da alta semelhança entre elas.

O grupo formado pela parcela quatro apresentou esse status de mais dissimilar, principalmente, pelo fato desta ter apenas um indivíduo (Figura 1c). Indivíduo este, que representou o maior volume $\left(1742.17 \mathrm{~m}^{3}\right)$ dentre todos aqueles obtidos pelas demais parcelas. Além desta, outras parcelas também compensaram sua baixa densidade com uma boa representatividade em suas médias de volume. Alexandre Junior \& Soares Júnior (2009), que obtiveram resultados semelhantes, justificam este fato como sendo consequência destas parcelas figurarem entre aquelas com as maiores concentrações de indivíduos de portes intermediários e grandes.

Deste modo, o volume surge como um parâmetro que pode ser visto, em determinados resultados, como o principal responsável pela formação dos agrupamentos. Fato comprovado pelo destaque de algumas poucas parcelas em relação às demais; mesmo estas, mostrando-se inferiores em abundância, porém, com maiores valores de altura e diâmetro.

No entanto, para confirmar a influência incisiva do volume na formação dos grupos, foi feita uma segunda análise, sem a presença do mesmo. Nesta análise foram encontrados seis grupos em nível de fusão 20, com as parcelas um, 18 e 28, compondo um grupo mais dissimilar aos demais (Figura 3). A distância destes grupos em relação aos demais foi determinada pela diferença da abundância por parcela, seguida pelas médias de altura e de diâmetro.

A forma com que diferentes povoamentos determinam as diferenças na paisagem foi salientada por Greig-Smith (1964), quando afirmou que a maior ou menor dissimilaridade entre grupos relativamente próximos, pode ser determinada pela reunião de parcelas cujas semelhanças entre si é comumente dada pela variação no número de indivíduos de uma parcela à outra, formando agrupamentos de parcelas semelhantes entre si quanto à densidade.

De forma complementar, no estudo de Hay et al. (2000) pode-se perceber o quão determinante pode ser a influência de um parâmetro de porte, como as alturas dos indivíduos, na dissimilaridade entre parcelas. São particularidades estruturais dentro de cada população como ravinas, voçorocas, afloramentos rochosos, entre outros, que resultam na fragmentação do seu espaço físico e estabelecem micro-ambientes.

A análise de ordenação por PCoA, a partir da planilha original, com os valores de volume inclusos, revelou que um único eixo (eixo um) é responsável por 99.56 \% (Figura 4) da variância entre os grupos formados. Variância determinada pela combinação entre as diferenças de volumes entre parcelas e o maior peso desses valores de volume em relação aos dos demais.

Assim, entendendo que mesmo após uma ponderação equilibrada dos valores avaliados, o volume continuaria a mascarar as designações dos eixos ao mesclar outros parâmetros já envolvidos na análise, optou-se por retirá-lo. O resultado da análise sem a presença do parâmetro volume revelou 74.94 \% dos agrupamentos determinados pelo mesmo eixo (eixo um) (Figura 5).

A análise mais criteriosa do gráfico de ordenação evidencia que a parcela quatro, se firmou como a mais distante das outras. Enquanto que, sobre as demais, a homogeneidade dos seus parâmetros é alta a ponto de não permitir que a análise aqui utilizada, de baixa robustez, discrimine grupos fracamente estabelecidos.

A análise de agrupamento para perfilho foi feita utilizando-se o Método de Ligação Completa (Máxima); pois o mesmo foi o que apresentou a maior Correlação Cofenética (C.Cf.=0.730747). Este método é baseado na máxima distância entre grupos, fundindo-se inicialmente aos menos distantes (Ferreira, 1996). Com ele foram formados dois grandes grupos quanto ao perfilhamento, até o ponto de fusão 17 (Figura 6). Isso indica que uma variável específica, aqui não identificada, determinou a formação destes grupos entre as parcelas. As maiores semelhanças entre parcelas vizinhas em um pequeno trecho, como as do presente estudo, são explicadas por Ribas et al. (2003), como sendo uma resposta à maior proximidade espacial entre elas; proximidade esta, que justifica a submissão de duas ou mais parcelas a um mesmo histórico de perturbação e de regeneração. 


\section{Conclusão.}

A presença de micro-ambientes inóspitos a maioria das espécies lenhosas conhecidas, porém, propensa ao desenvolvimento da espécie estudada, cria certa homogeneidade no espaço físico ocupado, favorecendo a tênue ligação entre as parcelas e agrupamentos destas. Uma homogeneidade territorial que só não deixa evidente a eficiência e facilidade de L. pinaster em explorar tal ambiente, distribuindo os seus indivíduos em grupos bem estabelecidos, de jovens a adultos; como também, a forma como esta se sobressai a muitas outras espécies, da mesma forma de vida, comuns no Cerrado Stricto Sensu das adjacências.

Dessa forma, além dos agrupamentos terem sido determinados pelas altas similaridades dos padrões estruturais, característicos da espécie, pela área de estudo; o histórico de ocupação, assim como de degradação e de regeneração, certamente tem influencia na maneira homogênea com que os 3000 $\mathrm{m}^{2}$ estudados abrigam sua cobertura vegetal.

Os estudos de como as populações vegetais se estabelecem em ambientes extremos e de grande endemismo, como os Campos Rupestres, constituemse em uma ferramenta crucial para a redução da perda causada pelo extrativismo predatório e intenso, lhes propiciando assim, maior proteção e conservação. Neste contexto, diante da representativa abundância encontrada, fica claro que o pousio e a exploração racional são as melhores estratégias para assegurar a sobrevivência das suas populações.

\section{Literatura citada}

Almeida S.P., Proença C.E.B., Sano S.M \& Ribeiro J.F. 1998. Cerrado: espécies vegetais úteis. Embrapa-Cpac, Planaltina.

Alexandre-Junior W.R \& Soares Júnior F.J. 2009. Estrutura populacional de Roupala montana Aubl. em um trecho de cerrado Sensu stricto no sul de Minas Gerais, Brasil. Pesquisas, Botânica (São Leopoldo-RS). 60: 301-314.

CETEC - Centro Tecnológico de Minas Gerais. 1983. Diagnóstico ambiental do Estado de Minas Gerais. Belo Horizonte: Fundação Centro Tecnológico de Minas Gerais/CETEC. Série de Publicações Técnicas/SPT010.

Coile N.C \& Jones S.B. 1981. Lychnophora (Compositae: Vernonieae), a endemic genus to the Brazilian planalto. Brittonia. 33:528-542.

COPAM - Conselho de Política Ambiental. 2009. Lista das espécies ameaçadas de extinção da flora do Estado de Minas Gerais. Deliberação COPAM 85/97.

Dantas A.A.A., Carvalho L.Z. \& Ferreira E. 2007. Classificação e tendências climáticas em Lavras. Ciências agrotécnicas (Lavras). 31(6):1862-1866.

Eidt R.C. 1968. The climatology of South América. En: Fittkau E.J., Illies J., Klinge H., Schwabe G.H. \& Sioli J.C.H (eds.). Biogegraphy and ecology in South America: 54-81. Dr. W. Junk N.V. Publishers, The Hague, Netherlands.
Ferreira D.F. 1996. Análise multivariada. Ministério da Educação e do Desporto. Universidade Federal de Lavras, Departamento de Ciências Exatas, Lavras-MG.

Fielder N.C., Carvalho I.N.C., Rezende A.V., Medeiros M.B. \& Venturoli F. 2004. Efeito de inscêndios florestais na estrutura e composição florística de uma área de cerrado Strictu sensu na Fazenda Água LimpaDF. Revista Árvore (Viçosa-MG). 28:129-138.

Greig-Smith M.A.P. 1964. Quantitative plant ecology. 3 ed. Blackwell. Oxford.

Harley R. M. 1995. Introduction. Páginas 43-78. En: STANNARD B. (editor.). Flora of the Pico das Almas, Chapada Diamantina, Bahia, Brazil. Royal Botanic Gardens, Kew.

Hay J.D., Bizerril M.X., Calouro A.M., Costa E.M.N., Ferreira A.A., Gastal M.L.A., Goes Junior C.D., Manzan D.J., Martins C.R., Monteiro J.M.G., Oliveira S.A., Rodrigues M.C.M., Seyffarth J.A.S. \& Walter B.M.T. 2000. Comparação do padrão da distribuição espacial em escalas diferentes de espécies nativas do cerrado, em Brasília, D.F. Revista Brasileira de Botânica (São Paulo). 23(3):341-347.

Harper J.L. 1977. Population biology of plants. Academic Press, New York.

Magalhães W.C.S., Missagia R.V., Costa F.A.F. \& Costa M.C.M. 2008. Diversidade de fungos endofíticos em Candeia Eremanthus erythropapus (DC.) MacLeish. Cerne (Lavras). 14(3):267-273.

Myers N. R. A., Mittermeier C. G., Mittermeier G. A. B. \& Fonseca J. K. 2000. Biodiversity hotspots for conservation priorities. Nature. 403:853-858.

Oliveira-Filho A.T. \& Fluminhan-Filho M. 1999. Ecologia da vegetação do Parque Florestal Quedas do Rio Bonito. Cerne. 5(2): 51-64.

Pereira M. C. A. 1994. Estrutura das comunidades vegetais de afloramentos rochosos dos campos rupestres do Parque Nacional da Serra do Cipó, MG. Dissertação (Mestrado em Ecologia)- Universidade Federal de Minas Gerais, Belo Horizonte.

Queiroz R., Souza A.G.., Santana P., Antunes F.Z. \& Fontes M. 1980. Zoneamento agroclimático do Estado de Minas Gerais. Secretaria da Agricultura, Belo Horizonte.

Ratter J.A., Ribeiro J.F. \& Bridgewater S. 1997. The brazilian cerrado vegetation and threats to its biodiversity. Annals of Botany. 80: 223-230.

Ribeiro J.F. 1983. Comparação da concentração de nutrientes na vegetação arbórea e nos solos de um cerrado e um cerradão no Distrito Federal, Brasil. MSc Thesis, University of Brasília, Brasil.

Ribeiro J.F. \& Walter B.M.T. 1998. Fitofisionomias do bioma cerrado. En: S.M. Sano.; S.P. Almeida (eds.). Cerrado: ambiente e flora: 89-166. Embrapa-CPAC, Planaltina.

Ribas R.F., Neto J.A.A.M., Silva A.F. \& Souza A.L. 2003. Composição florística de dois trechos em diferentes etapas serias de uma floresta estacional semidecidual em Viçosa, Minas Gerais. Revista Árvore (Viçosa). 27 (6): 821-830.

Rizzini C.T. 1992. Tratado de Fitogeografia do Brasil: aspectos ecológicos, sociológicos e florísticos. Âmbito Cultural, São Paulo.

Rodrigues V.E.G.. \& Carvalho D.A. 2001. Plantas medicinais no domínio do cerrado. UFLA, Lavras. 
Safford H.D. 1999. Brazilian Páramos I: Na introduction to the physical environment and vegetation of the campos de altitude. Journal of Biogeography (Amsterdam). 26: $693-712$.

Semir J. 1991. Revisão Taxonômica de Lychnophora Mart. (Vernonieae: Compositae). Tese de Doutorado em Biologia Vegetal-UNICAMP, Campinas.

Shepherd G.J. 1995. Fitopac 1: Manual de usuário. Departamento de Botânica, UNICAMP, Campinas.

Silva S.M.P. 1998. Arnica de campos rupestres Lychnophora pinaster Mart. Asteraceae - aspectos da fenologia e da germinação de aquênios. Páginas 1-18. En: UNESP 2. Plantas medicinais aromáticas e condimentares: avanços na pesquisa agronômica. São Paulo.

Souza A.V., Pinto J.B.P., Bertolucci S.K.Z., Corrêa R.M. \& Castro E.M. 2003. Germinação de embriões e multiplicação In Vitro de Lychnophora pinaster Mart. Ciências Agrotécnicas. Edição especial: 1532-1538.

Van Der Hammen T. 1983. The palaeoecology and palaeography of savannas. In: Bourlière F. (ed). Tropical savannas. Elsevier, Amsterdam: 19-35.

Veloso H. P., Rangel Filho A. L. R. \& Lima J. C. A. 1991. Classificação da vegetação brasileira, adaptada a um sistema universal. IBGE, Departamento de Recursos Naturais e Estudos Ambientais, Rio de Janeiro.

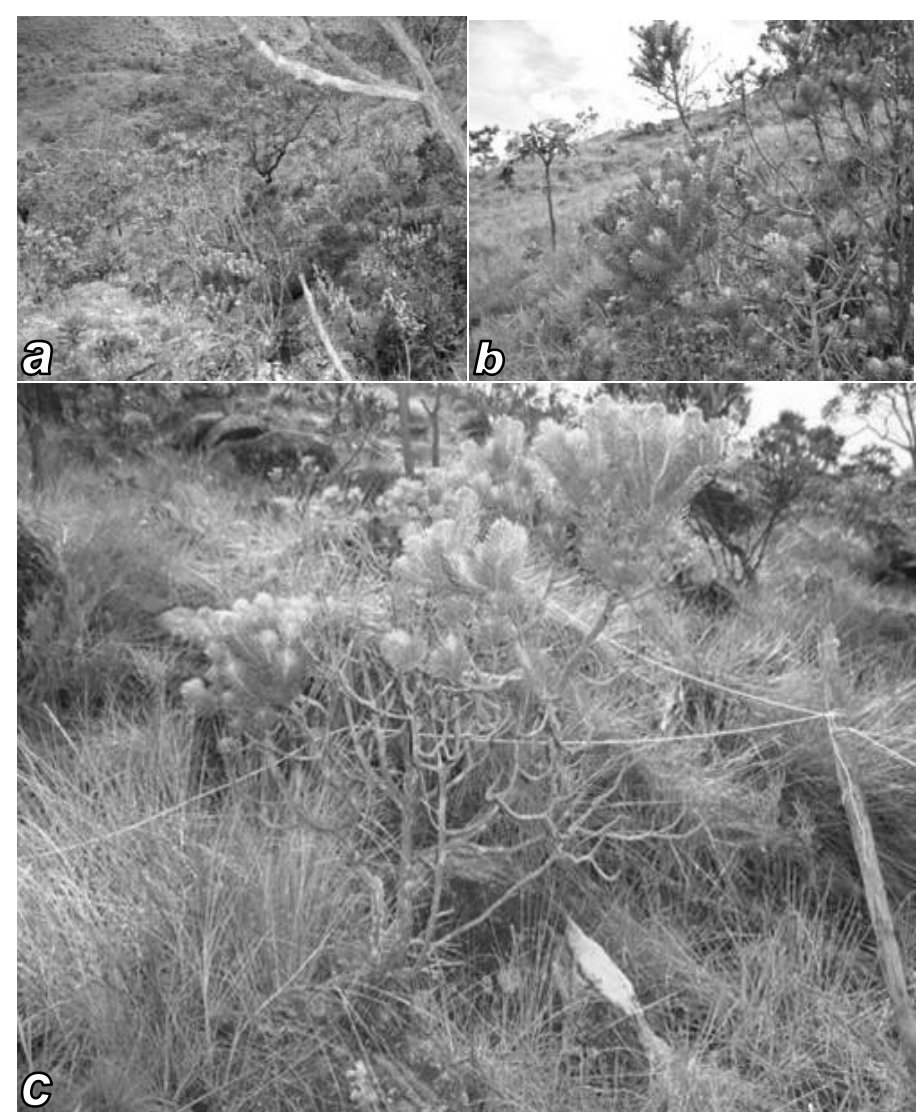

Figura 1. a) Trecho estudado de Campo Rupestre, na Reserva Biológica UNILAVRAS-Boqueirão, Ingaí, Minas Gerais; com b) Detalhes de um indivíduo florido de Lycnhophora pinaster dentro da área amostrada e c) do único indivíduo de Lychnophora pinaster encontrado na parcela quatro. 


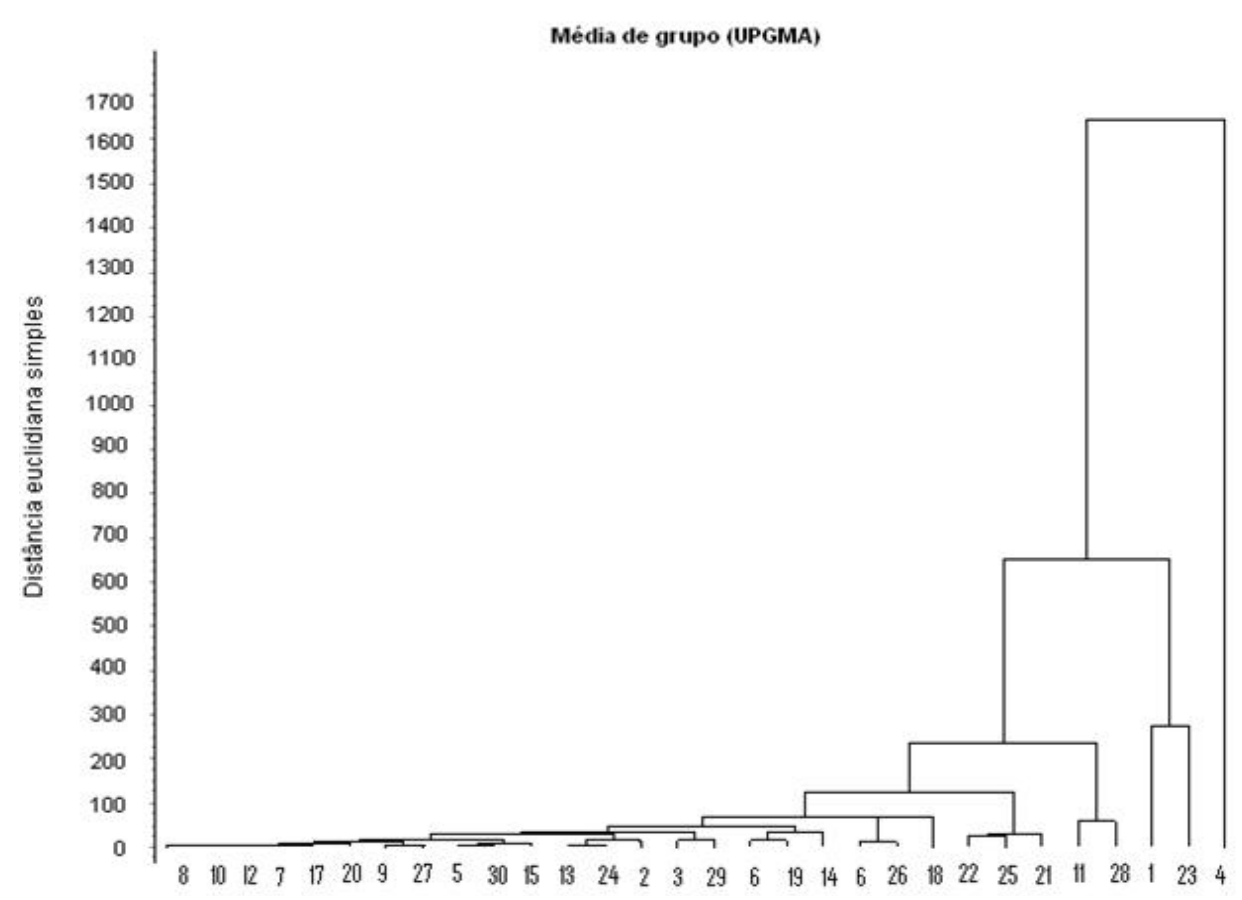

Figura 2. Dendrograma dos agrupamentos obtidos pela Análise de Médias de Grupos não Ponderadas (UPGMA) evidenciando os níveis de dissimilaridade encontrados entre as 30 unidades amostrais de uma população de Lychnophora pinaster na Reserva Biológica UNILAVRAS-Boqueirão, Ingaí, Minas Gerais.

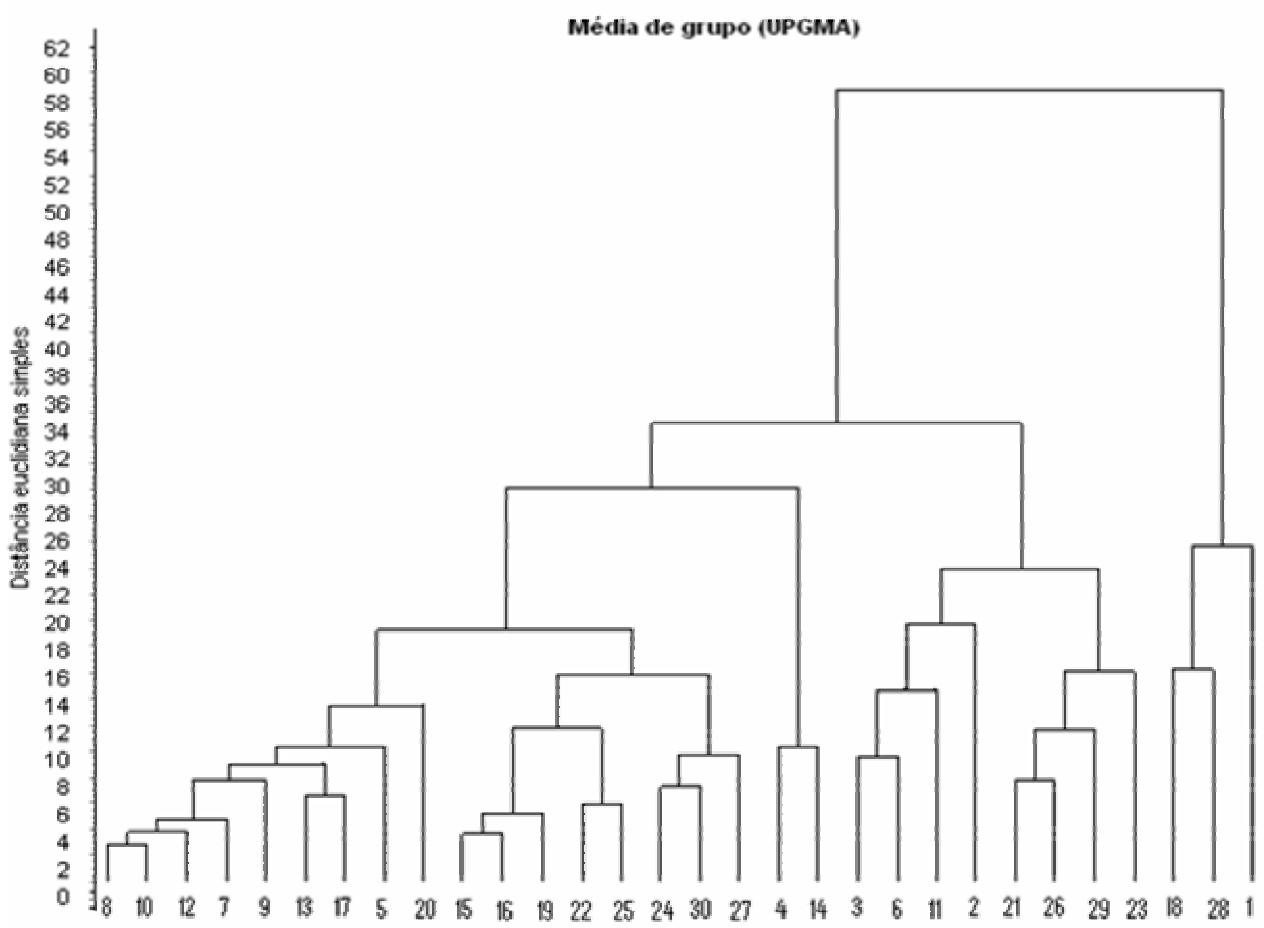

Figura 3. Dendrograma dos agrupamentos obtidos por média de grupo (UPGMA), sem presença do parâmetro volume, exibindo a dissimilaridade entre as 30 unidades de amostra de uma população de Lychnophora pinaster, na Reserva Biológica UNILAVRAS-Boqueirão, Ingaí, Minas Gerais. 


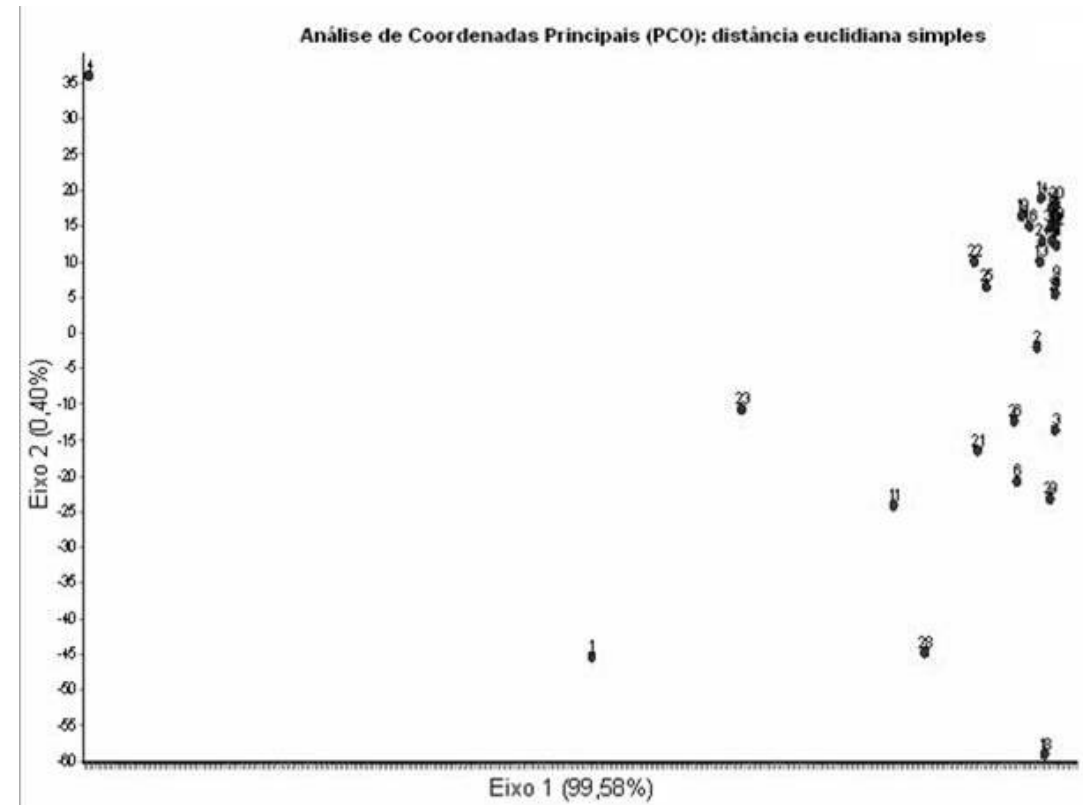

Figura 4. Análise em Coordenadas Principais (PCoA) evidenciando a ordenação dos grupos estabelecidos pelas 30 unidades de amostra de uma população de Lychnophora pinaster, na Reserva Biológica UNILAVRASBoqueirão, Ingaí - MG.

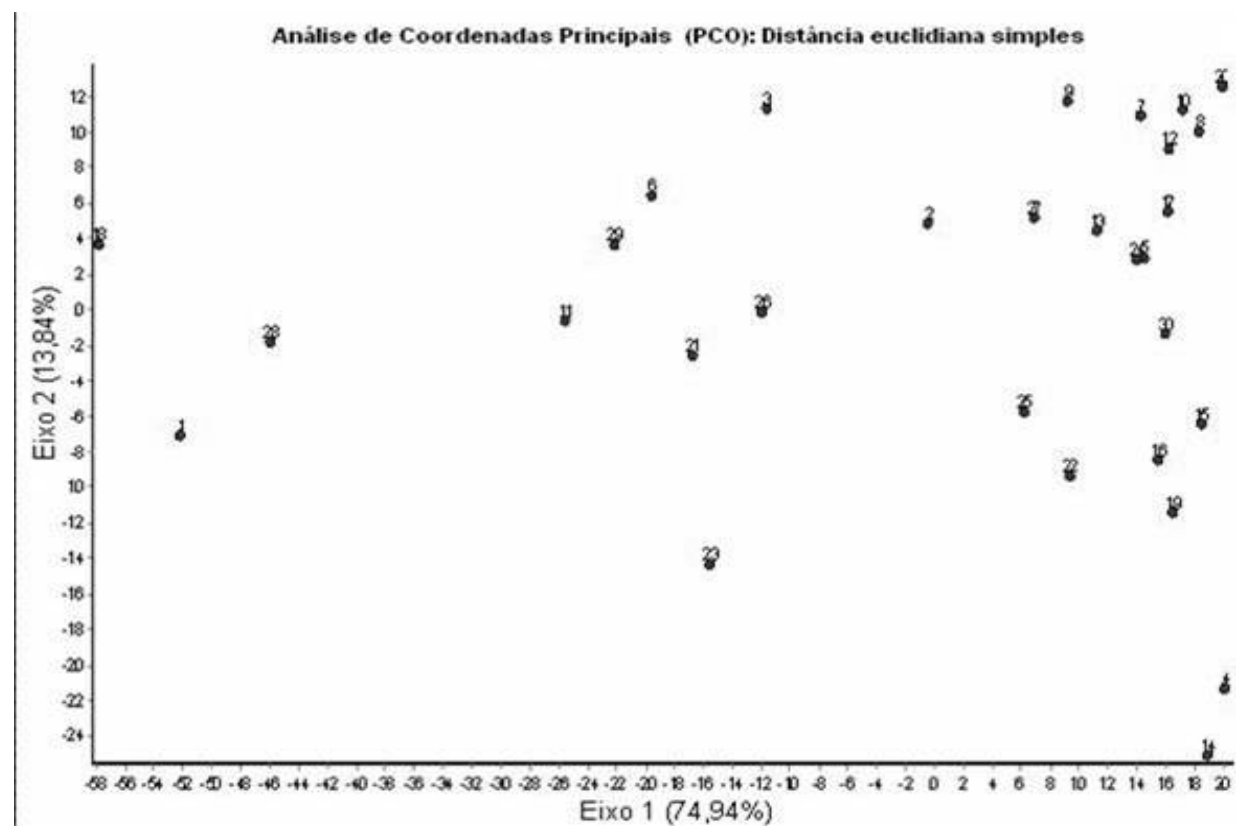

Figura 5. Análise em Coordenadas Principais (PCoA), sem a presença do parâmetro volume, exibindo a ordenação dos grupos estabelecidos pelas 30 unidades de amostra de uma população de Lychnophora pinaster, na Reserva Biológica UNILAVRASBoqueirão, Ingaí, Minas Gerais. 


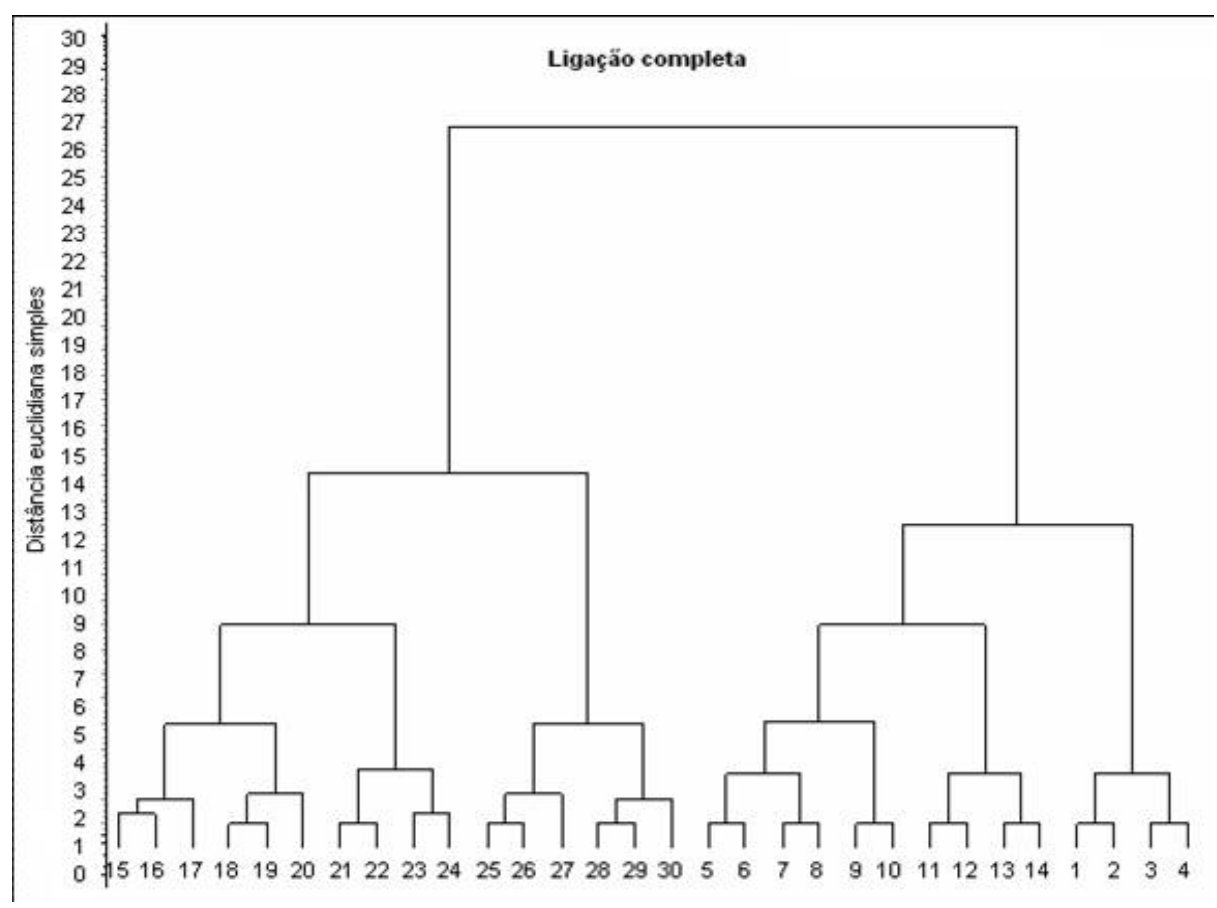

Figura 6. Dendrograma dos agrupamentos obtidos por Ligação completa, exibindo a dissimilaridade entre as 30 unidades de amostra de uma população de Lychnophora pinaster, pelo grau de perfilhamento, na Reserva Biológica UNILAVRAS-Boqueirão, Ingaí - MG.

\footnotetext{
${ }^{1}$ Centro Universitário de Lavras - UNILAVRAS, Rua Padre José Poggel, 506, CEP: 37200-000, Lavras - MG, Programa de Graduação em Ciências Biológicas (eciodiniz@yahoo.com.br)

${ }^{2}$ Programa de Pós-Graduação em Ecologia Aplicada, Departamento de Ecologia, Universidade Federal de Lavras, Lavras, Minas Gerais, Brasil (ana.pavanelli.bio@hotmail.com ).

${ }^{3}$ Professor Adjunto do Curso de Ciências Biológicas; Curador do Herbário de Lavras (Herbário LUNA), Centro Universitário de Lavras (UNILAVRAS), Lavras, Minas Gerais, Brasil. Rua Padre José Poggel, 506. Bairro Centenário, CEP.37200-000 (fjsoaresjunior@unilavras.edu.br / fjsoaresjunior@yahoo.com.br).

Autor para correspondências.
} 